\title{
1 The APETALA2 transcription factor LSAP2 regulates seed shape in lettuce
}

2

3 Running title: LsAP2 regulates lettuce seed shape

4

5 Chen Luo*, Shenglin Wang*, Kang Ning, Zijing Chen, Yixin Wang, Jingjing Yang,

6 Meixia Qi, Qian Wang ${ }^{\dagger}$

7

8 Beijing Key Laboratory of Growth and Developmental Regulation for Protected

9 Vegetable Crops, Department of Vegetable Science, College of Horticulture, China

Agricultural University, Beijing 100193, China

*These authors contributed equally to this work.

${ }^{\dagger}$ Author for correspondence: wangq@cau.edu.cn

Date of submission: 2020/11/23

Number of tables: 0

Number of figures: 7

Number of supplementary tables: 6

Number of supplementary figures: 6

Number of supplementary data: 1

Word count: 4530 (Introduction: 840; Results: 2481; Discussion: 1145; Acknowledgments: 64)

\section{Highlight}

The APETALA2 transcription factor LsAP2 regulates lettuce seed shape via interacting with LsBP and repressing brassinosteroid biosynthesis and signaling. 


\section{9}

\section{Abstract}

Seeds are major vehicles of propagation and dispersal in plants. A number of transcription factors, including APETALA2 (AP2), play crucial roles during the seed development process in various plant species. However, genes essential for seed development and the regulatory networks during seed development remain unclear in lettuce. Here, we identified a lettuce AP2 (LsAP2) gene that was highly expressed at the early stages of seed development. LsAP2 knockout plants obtained by the CRISPR/Cas9 system were used to explore the biological function of LsAP2. Compared with wild-type, the seeds of the Lsap2 mutant plants had increased length and decreased width, and developed an extended tip at the seed top. After further investigating the seed structural characteristics of Lsap2 mutant plants, we proposed a new function of $L S A P 2$ in seed dispersal. Moreover, we identified several interactors of LsAP2. Our results showed that LsAP2 directly interacted with the lettuce homolog of BREVIPEDICELLUS (LsBP) and promoted the expression of LsBP. Transcriptome analysis revealed that LsAP2 might also be involved in brassinosteroid biosynthesis and signaling pathways. Taken together, our data indicate that LsAP2 has a significant function in regulating seed shape in lettuce.

Keywords: lettuce, APETALA2, seed shape, LsAP2, CRISPR/Cas9, achene, regulatory networks

8




\section{Introduction}

In angiosperms, a mature seed has three major components, embryo, endosperm, and seed coat. The seeds that store essential substances and contain genetic materials are the most important harvested organ in many plant species. Moreover, seed size and seed shape are crucial for plants to adapt during evolution, and seed size is also an important agronomic trait in crop domestication (Moles et al., 2005).

Lettuce (Lactuca sativa L.) belongs to the Asteraceae family and is one of the most important leafy vegetables worldwide. The seed structure of lettuce is different from the model plant Arabidopsis as the lettuce seed is actually an achene fruit. Therefore, the achene development integrates the processes of fruit and seed development. Achene has four major components, pericarp, integuments, endosperm and embryo. The pericarp is derived from the ovary walls, and the pericarp and integuments are tightly integrated to constitute the seed coat (Steinbrecher and Leubner-Metzger, 2017). Lettuce seeds are the vehicles of propagation and dispersal, and many traits have changed dramatically during lettuce domestication, including seed size, seed shattering, and seed dispersal (Zhang et al., 2017). A previous study showed that some lettuce MADS-box genes were highly expressed in seeds, and these genes may be involved in seed development (Ning et al., 2019). However, few studies have performed the gene function analysis and explored the regulatory networks associated with seed development in lettuce.

Seed development is initiated by double fertilization (Jiang and Köhler, 2012). The zygote, the fertilized central cell and the maternal integuments develop together into a complete seed. Thus, the seed growth is coordinately controlled by the growth of maternal and zygotic tissues (Sreenivasulu and Wobus, 2013). Seed development is a complicated process modulated by complex networks. Recent studies have identified various pathways that control seed development through maternal tissues, including the ubiquitin-proteasome pathway, G-protein signaling pathway, mitogen-activated protein kinase signaling pathway, phytohormone signaling and homeostasis pathway, as well as various kinds of transcriptional regulatory factors ( $\mathrm{Li}$ and $\mathrm{Li}, 2015$; $\mathrm{Li}$ et al., 
2019). Many genes involved in the seed development process have been studied extensively, and many key regulators of seed size and seed shape have been identified (Sreenivasulu and Wobus, 2013; Li et al., 2019).

APETALA2 (AP2) gene belongs to the euAP2 lineage, a subgroup of the large AP2/ERF gene family. Genes in the euAP2 lineage encode two highly conserved AP2 domains and contain a microRNA172 (miR172) binding site (Kim et al., 2006; Zumajo-Cardona and Pabon-Mora, 2016). AP2 gene encodes a transcription factor, and its roles in specifying floral organ identity and regulating the expression of floral homeotic genes are well known (Kunst et al., 1989; Bowman et al., 1991; Coen and Meyerowitz, 1991; Drews et al., 1991). Meanwhile, miR172 regulates the expression of AP2 gene primarily through translational inhibition (Aukerman and Sakai, 2003; Chen, 2004). The AP2 gene was first isolated in Arabidopsis, and AP2 activity was shown to be required during seed development (Jofuku et al., 1994). A mutation in the AP2 gene (ap2-1) in Arabidopsis had a pleiotropic effect on seed shape, which changed from the normal oblong shape to a number of aberrant shapes (Leon-Kloosterziel et al., 1994). AP2 was shown to act as an important negative regulator of seed development because ap2 mutants produce large seeds. AP2 acts maternally to determine seed weight and seed yield by coordinating the growth of embryo, endosperm, and maternal integuments (Jofuku et al., 2005; Ohto et al., 2005; Ohto et al., 2009). Petunia AP2A, an ortholog of Arabidopsis AP2, is also expressed in seeds, and it was found to restore the structural defect of seed epidermal cells in Arabidopsis ap2-1 mutant (Maes et al., 2001). Grain yield is an important agronomic trait in crop domestication. In rice, SUPERNUMERARY BRACT (SNB) encodes an AP2-like transcription factor (Lee et al., 2007), and SUPPRESSION OF SHATTERING1 (SSH1) was recently found to be an allele of SNB. The rice ssh1 mutant shows reduced shattering and has larger seeds and higher grain weight (Jiang et al., 2019). A genome-wide association study of grain length and grain width in rice also identified $S N B$ as a novel gene that negatively regulates grain size. The $S N B$ knockout plants show increased grain length, width, and weight (Ma et al., 2019). 
114 of its high specificity and efficiency in editing the genomes of humans, animals, and

115 plants (Cong et al., 2013; Shan et al., 2013; Kumar and Jain, 2015), and scientists

116 have applied the CRISPR/Cas9 system to precisely modify crop traits and accelerate

117 the breeding process (Botella, 2019; Chen et al., 2019). In this study, we identified a

118 lettuce $A P 2$ (LsAP2) gene that was highly expressed at the early stages of seed

119 development. Then, we used the CRISPR/Cas9 system to edit LsAP2 and found that

120 LsAP2 regulates lettuce seed shape. We also showed LsAP2-related regulatory

121 networks during seed development in lettuce. Transcriptome analysis identified the

122 differentially expressed genes (DEGs) between WT and Lsap2 mutant plants.

123 Moreover, LsAP2 might regulate seed shape through its effects on the brassinosteroid

124 (BR) biosynthesis and signaling pathways. 
Materials and methods

144

\section{Plant materials and growth conditions}

Lettuce cultivar S39 was used as wild-type (WT) in this study. The lettuce seeds were provided by Dr. Han from Beijing University of Agriculture (Han et al., 2016). The lettuce plants were grown in growth chambers under a photoperiod of 16-h light (200 $\mu \mathrm{mol} \mathrm{m} \mathrm{m}^{-2} \mathrm{~s}^{-1}$ ) at $25^{\circ} \mathrm{C}$ and 8 -h dark at $18^{\circ} \mathrm{C}$. When the fifth true leaf was fully expanded, the lettuce plants were transplanted to a greenhouse in the Science Park of China Agricultural University under standard greenhouse conditions.

\section{Phylogenetic analysis}

Lettuce AP2 genes were identified using BLASTp from the lettuce genome (Reyes-Chin-Wo et al., 2017). Lettuce sequences were downloaded from the CoGe genome database (https://genomevolution.org/coge/). Arabidopsis sequences were downloaded from The Arabidopsis Information Resource (TAIR; https://www.arabidopsis.org/index.jsp). Petunia sequences were downloaded from the Sol Genomics Network https://solgenomics.net/organism/Petunia_axillaris/genome). Sequences of tomato and rice were downloaded from the Phytozome genome database (https://phytozome.jgi.doe.gov/pz/portal.html). Sequences of maize and wheat were downloaded from the National Center for Biotechnology Information (NCBI; https://www.ncbi.nlm.nih.gov). The amino acid sequences were aligned using ClustalW (Larkin et al., 2007). The phylogenetic tree was constructed in MEGA 5 program using the neighbor-joining method with 1000 bootstrap replicates (Tamura et $a l ., 2011)$. The accession numbers of the used sequences are listed in Supplementary Table S1.

\section{RNA extraction, cDNA synthesis, and qRT-PCR}

Total RNA was extracted from different lettuce tissues using the Quick RNA isolation 
Kit (Huayueyang, China). The cDNAs were synthesized using the FastKing-RT SuperMix (Tiangen, China) according to the manufacturer's protocol. Subsequently, quantitative real-time PCR (qRT-PCR) assays were performed in the QuantStudio 6 Flex Real-Time PCR System (Applied Biosystems, USA) using TB Green Premix Ex Taq II (Takara, Japan). The qRT-PCR assays were performed with technical triplicates of three biological replicates. The expression data were normalized using LsPP2A-1 and LsTIP41 as reference genes (Sgamma et al., 2016). Relative expression levels were calculated using the $2^{-\Delta \Delta C T}$ method (Livak and Schmittgen, 2001). Primers used for qRT-PCR assays are listed in Supplementary Table S2.

\section{Construction of the lettuce transformation vectors}

To generate the Lsap2 mutants, pKSE401-LsAP2 was constructed using the previously described method (Xing et al., 2014). The single-guide RNAs (sgRNAs) were designed using the Cas-Designer Tool (Park et al., 2015; Liu et al., 2017). Cas-OFFinder was used to predict the potential off-target sites of Cas9 RNA-guided endonucleases (Bae et al., 2014; Liu et al., 2017). The $\beta$-glucuronidase (GUS) reporter gene under the control of $L S A P 2$ promoter was used to generate the pLsAP2:GUS construct. Briefly, the 2402-bp genomic fragment upstream of the $L s A P 2$ start codon was amplified by PCR using genomic DNA as the template. Then, the fragment was inserted into the HindIII-BamHI sites of the pBI121 vector to drive the GUS reporter gene. Primers used to generate the above constructs are listed in Supplementary Table S2.

\section{Agrobacterium-mediated transformation of lettuce}

Lettuce transformation procedure was improved from the previously described method (Chen et al., 2017). The detailed steps are as follows. Seeds of lettuce cultivar S39 were sterilized with $1.0 \%$ sodium hypochlorite for $10 \mathrm{~min}$ and rinsed with sterile water, then sown on MS medium (MS supplemented with 3\% sucrose and 0.7\% agar). The plates were incubated under a photoperiod of 16-h light $\left(200 \mu \mathrm{mol} \mathrm{m} \mathrm{m}^{-2} \mathrm{~s}^{-1}\right)$ at 
$25^{\circ} \mathrm{C}$ and 8 -h dark at $18^{\circ} \mathrm{C}$ for about 5 days. When the cotyledons were expanded, they were cut and incubated with Agrobacterium GV3101 carrying the desired plasmid for $10 \mathrm{~min}$ in MS liquid medium (1/2 MS supplemented with 3\% sucrose and $200 \mu \mathrm{mol} / \mathrm{L}$ acetosyringone). After incubation, the cotyledons were transferred to sterile filter papers to remove excess Agrobacterium. Then, the cotyledons were placed on MS co-cultivation medium (MS supplemented with 3\% sucrose, $0.7 \%$ agar, $0.1 \mathrm{mg} / \mathrm{L}$ 6-benzylaminopurine, $0.05 \mathrm{mg} / \mathrm{L} \alpha$-naphthaleneacetic acid, and $200 \mu \mathrm{mol} / \mathrm{L}$ acetosyringone) and incubated at $25^{\circ} \mathrm{C}$ in the dark for 2 days. Subsequently, explants were transferred to MS selection medium (MS supplemented with 3\% sucrose, $0.7 \%$ agar, $0.1 \mathrm{mg} / \mathrm{L}$ 6-benzylaminopurine, $0.05 \mathrm{mg} / \mathrm{L} \alpha$-naphthaleneacetic acid, $300 \mathrm{mg} / \mathrm{L}$ timentin, and $40 \mathrm{mg} / \mathrm{L}$ kanamycin monosulfate) and incubated under a cycle of 16-h light $\left(200 \mu \mathrm{mol} \mathrm{m} \mathrm{m}^{-2} \mathrm{~s}^{-1}\right)$ at $25^{\circ} \mathrm{C}$ and 8 -h dark at $18^{\circ} \mathrm{C}$. About two weeks later, regenerated shoots grew on the calli, and they were cut into MS shoot-inducing medium (MS supplemented with 3\% sucrose, 0.7\% agar, and $300 \mathrm{mg} / \mathrm{L}$ timentin). After two weeks, the shoots were subcultured on fresh MS shoot-inducing medium. When the shoots were 2-cm long, they were transferred to MS rooting medium $(1 / 2$ MS supplemented with $3 \%$ sucrose, $0.7 \%$ agar, $0.02 \mathrm{mg} / \mathrm{L} \alpha$-naphthaleneacetic acid, and $200 \mathrm{mg} / \mathrm{L}$ timentin). When the shoots and roots of the regenerated plants were well developed, they were transferred to the soil under standard growth conditions.

\section{GUS staining}

Tissues from the $p L s A P 2: G U S$ plants were used for GUS staining assays. First, flowers and seeds at different developmental stages were collected and immersed in GUS staining buffer (containing $1 \mathrm{mM} 5$-bromo-4-chloro-3-indolyl- $\beta$-D-glucuronide). Next, the samples were vacuum infiltrated for $10 \mathrm{~min}$, and then stained in a $37^{\circ} \mathrm{C}$ incubator overnight. After incubation, the staining buffer was removed, and samples were washed with $70 \%$ ethanol for several times to remove the pigments. Finally, the samples were immersed in fresh $70 \%$ ethanol before observation. The samples were observed using a Leica stereomicroscope (Leica S8 APO, Germany). 


\section{Phenotypic analysis}

225

226

227

228

229

230

231

232

Plant materials were photographed with a digital camera (Nikon D7000, Japan) and a stereomicroscope (Leica S8 APO, Germany). To evaluate seed size, the images were imported into ImageJ software to measure the seed length and seed width. The measured seed length and seed width were used to calculate the ratio of seed length to width. To obtain the scanning electron micrographs, the seeds were gold plated and observed using a scanning electron microscope (Hitachi S-3400N, Japan). To evaluate cell size, the images were imported into ImageJ software to measure the cell length and cell width. Mature seeds were evaluated for calculating 1,000-grain weight using an electronic analytical balance (METTLER TOLEDO, USA). For seed germination tests, 20 seeds were tested for each line with three replicates, then the seeds were moistened with water and incubated at $25^{\circ} \mathrm{C}$ for two days to calculate the germination rates. To measure the seed beak diameter, beaks were photographed using a microscope (Olympus IX71, Japan), then the images were imported into ImageJ software to calculate the beak diameter. To evaluate seed dispersal, the breaking tensile strength (BTS) between beak and seed was measured with a digital force gauge (SHSIWI, China) at the harvest stage.

\section{Yeast two-hybrid assays}

The full-length coding sequence (CDS) of LsAP2 was cloned into pGADT7 vector. The full-length CDSs of $L s A S 1, L s A S 2, L s B P, L s K A N 1$, and LsKAN2 were amplified and cloned into pGBKT7 vector. Yeast two-hybrid assays were performed as described previously (Ding et al., 2015). Briefly, bait plasmids and prey plasmids were co-transformed into yeast strain AH109 according to the manufacturer's protocol of GAL4-based yeast two-hybrid system (Clontech, USA). Then, yeast transformants were grown on SD/-Leu-Trp plates for growth of the colonies and grown on $\mathrm{SD}$-Leu/-Trp/-His/-Ade plates for protein interaction selection. Primers used for yeast two-hybrid assays are listed in Supplementary Table S2. 


\section{Firefly luciferase complementation imaging (LCI) assays}

252

253

254

255

256

257

258

259

260

261

262

263

264

265

266

267

268

269

270

271

272

273

274

275

276

The full-length CDSs without the stop codon of $L s A S 2, L s B P, L s K A N 1$, and LsKAN2 were cloned into the 35S: $n L U C$ vector. The full-length CDS of $L s A P 2$ was cloned into the 35S:cLUC vector. Then, the above constructs were transformed into Agrobacterium strain GV3101. LCI assays were performed using the previously described method (Chen et al., 2008). Briefly, different combinations were co-infiltrated into tobacco leaves using Agrobacterium-mediated transient transformation. After infiltration, the plants were grown under a 16-h light/8-h dark cycle for 2 days. Before imaging, the abaxial sides of the tobacco leaves were sprayed with luciferin, and the leaves were kept in the dark for $5 \mathrm{~min}$. Then a cooled CCD camera (Roper 1300B, USA) was used to capture the fluorescence signal with 10 min exposures. Primers used for LCI assays are listed in Supplementary Table S2.

\section{Dual-luciferase reporter assays}

The $L s B P$ promoter (1595-bp genomic fragment upstream the start codon) was cloned and inserted into the pGreenII 0800-LUC vector as the reporter. The full-length CDSs of $L s A P 2$ and $L s B P$ were inserted into the pGreenII 62-SK vector as effectors. The empty vector pGreenII 62-SK was used as the negative control. Dual-luciferase reporter assays were performed as described previously (Hellens et al., 2005). Briefly, Agrobacterium strain GV3101 carrying the reporter and effector constructs were infiltrated into tobacco leaves. After infiltration, the plants were grown under a 16-h light/8-h dark cycle for 2 days. Luciferase (LUC) and renilla luciferase (REN) activities were measured by a multiscan spectrum (Molecular Devices SpectraMax ${ }^{\circledR}$ i3x, USA) using the Dual-Luciferase Reporter Assay Kit (Promega, USA) according to the manufacturer's protocol. The ratio of LUC to REN was calculated as the final transcriptional activity. Primers used for dual-luciferase reporter assays are listed in Supplementary Table S2. 


\section{Transcriptome analysis}

278

279

280

281

282

283

284

285

286

287
Total RNA was extracted from the seeds of WT and Lsap2 mutant plants on the day of pollination, with three biological replicates. Paired-end libraries were constructed and sequenced using an Illumina NovaSeq 6000 platform at Biomarker Technologies (BioMarker, China). Analyses of the transcriptome data were performed on the BMKCloud platform (www.biocloud.net). Clean reads were mapped to the lettuce reference genome (Reyes-Chin-Wo et al., 2017) using the HISAT2 software (Kim et al., 2015). Differential expression analysis between WT and Lsap2 mutant plants (fold change $\geq 2$, FDR $<0.01$ ) was performed using the DESeq (Anders and Huber, 2010). Gene Ontology (GO) enrichment analysis and Kyoto Encyclopedia of Genes and Genomes (KEGG) analysis of the DEGs were performed using GOseq R packages and KOBAS software (Mao et al., 2005; Young et al., 2010).

\section{Measurement of endogenous hormones}

Extraction and purification of the endogenous hormones were performed as described previously (Wang et al., 2012). Briefly, about $0.2 \mathrm{~g}$ of the fresh seed samples from WT and Lsap2 mutant plants were collected and homogenized in $4 \mathrm{~mL}$ of $80 \%$ methanol (containing antioxidant), with three biological replicates. After incubation at $4^{\circ} \mathrm{C}$ for $48 \mathrm{~h}$, the extract was centrifuged at $5000 \mathrm{rpm}$ for $10 \mathrm{~min}$ at $4^{\circ} \mathrm{C}$. Then, the supernatant was passed through the extraction cartridge, and the phytohormone fraction was eluted with methanol and then ether. Next, the eluate was dried down and dissolved in phosphate-buffered saline (PBS) buffer. Quantification of auxin (IAA) and BR were measured by enzyme-linked immunosorbent assay (ELISA) following the described protocol (Zhao et al., 2006) at the Center of Crop Chemical Control, China Agricultural University. 


\section{Results}

\section{Identification of $A P 2$ genes in lettuce}

To understand the seed development process in lettuce and identify the key regulators during lettuce seed development, we continuously observed the developing seeds after pollination. We found that the seed morphology was fully developed within ten days after pollination (Fig. 1A). The seeds grew longer and wider in the first three days. Then, the seeds began to expand, and obvious longitudinal ribs were observed on the seed surface. One week later, the seed coat became darker, and the longitudinal ribs were more pronounced. Ultimately, the seeds were ready to ripen.

Previous studies have identified many transcription factors as important regulators of seed development (Li et al., 2019), and AP2 gene has been shown to play crucial roles in regulating seed development in many species. To investigate whether lettuce AP2 gene plays a role in seed development, we performed BLAST searches at the lettuce genome database and identified three lettuce $A P 2$ genes as the candidate genes. The phylogenetic tree revealed that LsAP2 was more closely related to Arabidopsis AP2 than the other two lettuce AP2 homologs, namely LsAP2-like1 and LsAP2-like2 (Supplementary Fig. S1).

To functionally characterize the lettuce $A P 2$ genes during seed development, we examined their expression patterns by analyzing the FPKM values from a transcriptome dataset. Strikingly, LsAP2 was the most highly expressed gene among the three lettuce $A P 2$ genes at different seed development stages, and the expression of LsAP2 gradually decreased between successive stages (Supplementary Table S3). According to the homology and expression analysis, we speculated that of the three lettuce $A P 2$ genes, $L S A P 2$ might be the one that plays a major role during lettuce seed development. Therefore, we chose $L s A P 2$ for further characterization in this study.

\section{Expression pattern of $L s A P 2$}

To detect the expression pattern of $L s A P 2$ further, we analyzed its expression in 
different lettuce tissues by qRT-PCR assays. We found that $L s A P 2$ was expressed in all lettuce tissues, and the expression level of $L s A P 2$ in flower was many times higher than in other tissues (Supplementary Fig. S2). Further, we examined the expression of $L s A P 2$ in floral organs. $L s A P 2$ was highly expressed in petal, pistil, and seed, while $L S A P 2$ displayed lower expression level in bract and stamen (Fig. 1B). To explore when LsAP2 was expressed during seed development, we then analyzed the expression of $L s A P 2$ in seeds at different developmental stages. Significantly, $L s A P 2$ transcripts were strongly accumulated at the early stages of seed development, and its expression decreased gradually as the seed developed (Fig. 1C). These results confirmed that $L S A P 2$ was expressed in seeds, especially at the early stages of seed development.

Further, we analyzed the spatial and temporal expression pattern of $L s A P 2$ in flower using the GUS reporter gene under the control of LsAP2 promoter. The observed GUS activities were generally consistent with the endogenous LsAP2 mRNA level determined by qRT-PCR. GUS staining showed that LsAP2 was mainly expressed in petal, pistil, and seed, while weaker or even no GUS signal was observed in bract and stamen (Fig. 1F-H). Then, we analyzed the expression of LsAP2 at different seed development stages. Strong GUS activities were also detected in seeds at the corresponding stages (Fig. 1J-M). Further, we observed different sections of the stained seeds, and only strong GUS signal was found in the seed coat (Fig. 1O-Q).

\section{CRISPR/Cas9-mediated editing of $L s A P 2$ alters seed shape}

To investigate the biological function of $L s A P 2$ gene, we generated $L s A P 2$ knockout plants using the CRISPR/Cas9 system (Fig. 2A). Genotyping analysis of the ten T0 transgenic plants identified six plants with mutations in the target sites. There were significant differences in the editing efficiency of the two targets; Target 1 was mutated in six plants, whereas Target 2 was mutated in only one plant (Supplementary Figs. S3, S4). Therefore, it is crucial to assess the on-target efficiency when design sgRNAs. There were two types of mutations among the mutant plants; two plants 
357 were homozygous, and four plants were biallelic that had two mutant alleles

358 (Supplementary Table S4). We also assessed the potential off-target sites using 359 Cas-OFFinder, and no mutation was detected in the predicted off-target sites 360 (Supplementary Table S5). Through the self-pollination of lettuce, homozygous and 361 non-transgenic T1 generation plants were obtained and confirmed by PCR genotyping 362 and sequencing. Three mutant lines were chosen for further exploration (Fig. 2B, C).

363 Given that the CRISPR/Cas9 system was used to edit the genomic sequence of $364 L s A P 2$, genome editing resulted in the non-sense transcripts of $L s A P 2$ gene. These 365 transcripts disrupted the normal translation of LsAP2 mRNA with frame-shift 366 mutation and premature stop codons (Supplementary Data S1), which led to the 367 absence of functional LsAP2 protein. AP2 is an A-class gene that determines perianth 368 identity in flowers (Kunst et al., 1989; Coen and Meyerowitz, 1991). However, we 369 did not observe any defect in the bract, petal, or pappus of Lsap2 mutant plants. 370 Interestingly, we found that the seed shape was changed in all mutant lines because 371 the seeds were lanky, and there was an extended tip at the top of the seed (Fig. 2D).

372 In Lsap2 mutant plants, the seed length increased significantly (Fig. 2E, H), and the 373 seed width decreased significantly (Fig. 2F, I), whereas the 1,000-grain weight did not 374 change significantly compared with WT (Fig. 2J). Seed germination tests showed that 375 almost all seeds germinated, and the roots developed normally after two days 376 incubation at $25^{\circ} \mathrm{C}$ (Fig. $2 \mathrm{G}, \mathrm{K}$ ), which indicated that the seed germination was not 377 affected in Lsap2 mutant plants. The scanning electron micrographs revealed that the 378 seed surface of Lsap2 mutant plants was smoother, and there were fewer longitudinal 379 ribs and striking spines on the seed surface of Lsap2 mutant plants compared with WT 380 (Supplementary Fig. S5). Further, the length of the seed epidermal cells in Lsap2 381 mutant plants was slightly longer than in WT, while the cell width did not change 382 significantly (Supplementary Fig. S5), indicating that the elongated cell length might 383 lead to the increased seed length in Lsap2 mutant plants. 


\section{Important roles of $L s A P 2$ in regulating seed morphology}

As LsAP2 was highly expressed at the early stages of seed development, we speculated that LsAP2 might regulate seed shape at the early stages. To verify this idea, we observed the seeds of WT and Lsap2 mutant plants at different seed development stages. Compared with WT, the seeds of Lsap2 mutant plants were already longer on the day of pollination (Fig. 3A). Subsequently, the seeds of Lsap2 mutant plants grew longer and thinner than those of the WT, while the seed beak of Lsap2 mutant plants was shorter and thicker than that of the WT (Fig. 3B-D). Because the seeds of Lsap2 mutant plants were lanky, we measured the length-width ratio of the mature seeds and showed that Lsap2 mutant plants had a larger seed length-width ratio compared with WT (Fig. 3E). Interestingly, in Lsap2 mutant seeds, the lower part of the beak and upper part of the seed seemed to be fused (Fig. 3B-D). After seed ripening, the beaks were broken and seeds were harvested, and an extended tip was left at the top of the Lsap2 mutant seeds. These results confirmed that the seed shape of Lsap2 mutant plants was altered at the early stages of seed development, and this phenotype corresponded to the high expression of $L s A P 2$ at these stages.

In Lsap2 mutant seeds, the beak was thicker and closely connected with the seed, suggesting that this structure might resist stronger external force and contribute to seed dispersal. To verify this hypothesis, we first measured the diameter of the seed beak at the harvest stage, and our data showed that the seed beak diameter of Lsap2 mutant plants was larger than that of the WT (Fig. 3F). Further, we measured the breaking tensile strength (BTS) between beak and seed, and the results showed that Lsap2 mutant plants had stronger BTS than WT (Fig. 3G), implying that LsAP2 might have a function in seed dispersal.

\section{LsAP2-related protein interaction networks during lettuce seed development}

Previous studies have shown that AP2 regulates genes related to fruit and seed development. However, whether AP2 could directly interact with these regulators to modulate fruit or seed development are largely unknown. To identify the proteins that 
412 potentially interact with LsAP2, we performed yeast two-hybrid assays to test the 413 interactions between LsAP2 and regulators associated with fruit and seed 414 development. Our results showed that LsAP2 directly interacted with four proteins, 415 namely the lettuce homologs of ASYMMETRIC LEAVES2 (AS2), 416 BREVIPEDICELLUS (BP), KANADI1 (KAN1), and KANADI2 (KAN2) (Fig. 4A). 417 AS2 encodes a LOB domain protein, and AS2 is required for leaf and fruit 418 development in Arabidopsis (Iwakawa et al., 2002; Alonso-Cantabrana et al., 2007). $419 B P$ is a class I KNOX gene (Venglat et al., 2002), which modulates replum formation 420 during fruit development in Arabidopsis (Alonso-Cantabrana et al., 2007). KAN1 and 421 KAN2 are members of the KANADI (KAN) family, and KAN1 and KAN2 have been 422 shown to play important roles in the growth of ovule integuments (McAbee et al., 423 2006).

424 To confirm these potential interactions in planta, we performed LCI assays in tobacco leaves. Co-expression of LsAP2 with LsAS2, LsBP, or LsKAN1 produced strong fluorescence activity (Fig. 4B), whereas co-expression of LsAP2 with LsKAN2 or control vectors showed only background levels of fluorescence activity (Fig. 4B). These results demonstrated that LsAP2 could interact with LsAS2, LsBP, and LsKAN1 in planta.

430 To determine whether the interactions of LsAP2 with LsAS2, LsBP, and LsKAN1 431 might be important for seed development, we performed expression analyses of $L s A S 2, L s B P$, and $L s K A N 1$. Our data showed that $L s B P$ and $L s K A N 1$ were expressed 433 in seeds, while $L s A S 2$ was not detected in seeds. Significantly, LsBP was strongly expressed in seeds (Supplementary Fig. S6). Further experiments were performed to examine the expression levels of $L s B P$ and $L s K A N 1$ at different seed development stages. The qRT-PCR analysis showed that $L s B P$ was expressed at all stages and displayed higher expression level at the early stages, and the expression of $L s B P$ decreased with the development of the seeds (Fig. 4C), which was overlapped temporally with the expression pattern of $L s A P 2$. The transcripts of $L s K A N 1$ could also be detected at different developmental stages, but LsKANl exhibited a different expression pattern from $L s A P 2$ (Fig. 4C). The biological relevance between $L s A P 2$ 
and $L s B P$ indicated that LsAP2 might cooperate with LsBP to regulate seed shape.

\section{LsAP2 affects the expression of fruit development related genes}

During the fruit development of Arabidopsis, AP2 negatively regulates $B P$ and REPLUMLESS $(R P L)$ to prevent replum overgrowth. Meanwhile, AP2 negatively regulates valve margin formation by repressing the expression of valve margin identity genes (Ripoll et al., 2011). Since the seeds of lettuce are also fruits, and the morphology of Lsap2 mutant seeds was changed, we then examined the expression of fruit development related genes in Lsap2 mutant plants. Compared with WT, the expression levels of $L s B P$ and $L s R P L$ were significantly decreased in the seeds of Lsap2 mutant plants (Fig. 5A). However, orthologs of SHATTERPROOF1 (SHP1), SHATTERPROOF2 (SHP2), INDEHISCENT (IND), and ALCATRAZ (ALC), which are required for valve margin formation and dehiscence, had not been found in lettuce.

The phylogenetic analysis revealed that LsAP2 was closely related to Arabidopsis AP2, and we demonstrated that LsAP2 also functioned in lettuce seed and fruit development. However, our results implied that LsAP2 might positively regulate $L s B P$ and $L s R P L$, which was opposite from Arabidopsis. Actually, the fruit structure of lettuce is different from that of Arabidopsis. The achenes of lettuce are indehiscent fruits, whereas the siliques of Arabidopsis are dehiscent fruits. Similarly, the caryopses of rice are also dry fruits with indehiscent, and the AP2-like transcription factor SNB positively regulates the expression of two rice $R P L$ orthologs, $q S H 1$ and SH5 (Jiang et al., 2019). These results indicated that the AP2 genes might exhibit divergent regulatory patterns between different types of fruit because of the structural differences.

Interestingly, LsAP2 interacted with LsBP at the protein level, and the LsBP gene was also down-regulated in Lsap2 mutant plants. We speculated that LsBP or LsAP2 might promote $L s B P$ expression, and the protein interaction between LsAP2 and LsBP would enhance the promotion. To test this regulatory network, we used the $L S B P$ promoter to drive $L U C$ reporter gene and performed dual-luciferase reporter 
assays. Compared with the control experiment, expression of LsAP2 or LsBP alone had a minor effect on the promotion of LUC activity, while co-expression of LsAP2 and LsBP significantly increased the LUC activity (Fig. 5C), indicating that LsAP2 cooperated with LsBP to enhance the activity of $L s B P$ promoter. These results demonstrated that the protein interaction between LsAP2 and LsBP could promote the expression of $L s B P$, which might be important for seed development in lettuce.

\section{Identification of differentially expressed genes between WT and Lsap2 mutant plants}

To dissect the molecular function of $L s A P 2$ in seed development further, we performed the transcriptome analysis of the seeds from WT and Lsap2 mutant plants on the day of pollination. Many genes were differentially expressed between WT and Lsap2 mutant plants, and we identified 586 up-regulated genes and 547 down-regulated genes among the transcriptomes (Supplementary Table S6). GO enrichment analysis of the DEGs revealed that they were involved in multiple processes. Significantly enriched GO terms included nucleosome assembly and its related cellular component. Moreover, some GO categories were related to photosynthesis (Fig. 6A). Nucleosome assembly is involved in DNA replication and cell division, and seed photosynthesis is essential for storage activity. These processes are necessary for normal seed development.

Further KEGG analysis of the DEGs indicated that some hormone-related genes were differentially expressed between WT and Lsap2 mutant plants, such as cytokinin, auxin, and BR. Phytohormones have various functions in plant growth and development. Because auxin and BR have been shown to regulate seed growth (Jiang et al., 2013; Figueiredo and Köhler, 2018), we measured the endogenous hormones in the developing seeds of WT and Lsap2 mutant plants. We found that the BR level was significantly increased in the developing seeds of Lsap2 mutant plants compared with WT. However, there was no significant difference in IAA level between WT and Lsap2 mutant plants (Fig. 6B). 
498 To explore the significance of the increased BR level in Lsap2 mutant seeds, we 499 examined the expression of BR-related genes that have been shown to control seed

500 development. The transcriptome data indicated that a lettuce homolog of 501 BRASSINOSTEROID INSENSITIVE 1-ASSOCIATED KINASE 1 (BAK1) was 502 significantly up-regulated in Lsap2 mutant plants (Fig. 6C), while the expression 503 levels of other BR-related genes were not changed significantly. We also performed 504 qRT-PCR assays to verify the transcription levels of some genes, and the results were 505 generally consistent with the transcriptome data (Fig. 6D). BAK1 is a receptor kinase 506 involved in BR signal transduction ( $\mathrm{Li}$ et al., 2002), and it has been shown to regulate 507 grain size in rice. Loss of function of $O s B A K 1$ exhibits decreased grain size (Yuan et 508 al., 2017). Thus, we speculated that the increased BR level in Lsap2 mutant seeds 509 might stimulate the BR perception and signaling, thus promoting cell elongation and 510 increasing seed length. 


\section{Discussion}

\section{Unique roles of $L S A P 2$ during seed development in lettuce}

$A P 2$ gene encodes a transcription factor, and the broad roles of the $A P 2$ gene in plant growth and development have been shown in many species. Here, we identified a lettuce $A P 2$ gene and explored its biological function. Expression analysis revealed that LsAP2 was highly expressed in seeds, especially at the early stages of seed development (Fig. 1C). We used the CRISPR/Cas9 system to edit LsAP2 gene and found that LsAP2 regulates lettuce seed shape (Fig. 2A-D). Although the loss of AP2 function alters seed shape in both Arabidopsis and lettuce, there are some differences. In Arabidopsis, ap 2 mutants produce large seeds with aberrant shapes. Reducing AP2 gene activity increases seed length and width and leads to increased seed mass (Jofuku et al., 2005; Ohto et al., 2005). Meanwhile, the epidermal cells of ap2 mutant seeds lack the columella structure, and the epidermal cells are larger and more irregular in shape than WT seeds (Jofuku et al., 1994). In lettuce, the seed shape of Lsap2 mutants was affected as the seeds were lanky and developed an extended tip (Fig. 2D-F). In Lsap2 mutant plants, the seed length increased, and the seed width decreased (Fig. 2H, I), while the seed mass did not change significantly compared with WT (Fig. 2J). Our scanning electron micrographs showed that the seed surface of Lsap2 mutants was smoother than that of the WT due to decreased longitudinal ribs and fewer striking spines. Moreover, cell length of the seed epidermal cells increased slightly in Lsap2 mutant plants, while the cell shape and cell structure did not show noticeable changes (Supplementary Fig. S5).

In the Asteraceae plant dandelion, the pappus attaches to the beak for seed dispersal, and the seed falls and stops dispersing when the junction between beak and seed is broken. Lettuce also belongs to the Asteraceae family and has the same seed structure as dandelion. In Lsap2 mutant seeds, the beak was thicker than that of the WT (Fig. 3A-D). Besides, the beak was closely connected with the seed top, and the BTS between beak and seed was stronger than WT (Fig. 3G), implying that this structure 
may resist stronger external force and contribute to longer distance seed dispersal with the help of the wind. This finding may also reveal a potential new function of LsAP2 in seed dispersal, which is radically divergent from Arabidopsis as the seed structure of lettuce is different from Arabidopsis.

Since $A P 2$ is an A-class gene that determines the identity of perianth organs, the sepals and petals transform into reproductive organs in ap2 mutants (Kunst et al., 1989; Coen and Meyerowitz, 1991). Although the expression analysis showed that $L S A P 2$ was expressed in bract and petal (Fig. 1B), we did not observe any perianth defect or transformation in the floral organs of Lsap2 mutant plants. On one hand, the petunia AP2-type REPRESSOR OF B-FUNCTION (ROB) genes have incomplete A-function. The $R O B$ genes are redundantly required for the normal development of the perianth and are not required to antagonize the C-function in the perianth (Morel et al., 2017). Based on current understanding, it is also possible that the complete A-function of $A P 2$ gene does not occur in lettuce. On the other hand, the lettuce reference genome was published in 2017, and researchers found that some types of genes, including $A P 2$, were enriched in the triplicated regions (Reyes-Chin-Wo et al., 2017). Indeed, there are three $A P 2$ genes in lettuce (Supplementary Fig. S1), and this gene redundancy mechanism may explain the absence of perianth defect in Lsap2 mutant plants.

\section{LsAP2 may regulate seed shape via interacting with LsBP}

In Arabidopsis, the regulatory networks of fruit development have been studied extensively, and many genetic regulators involved in fruit development were identified. BP and RPL play direct roles in promoting replum identity during fruit development. AP2 cooperates with AS1 and AS2 to modulate replum development by independently regulating the activity of $B P$ and $R P L$ (Ripoll et al., 2011). Although their interactions are known at the genetic level, the direct protein interaction networks of AP2 in fruit or seed development are largely unknown. In this study, we identified several interactors of LsAP2 using yeast two-hybrid and LCI assays. LsAP2 
583 directly interacted with LsBP in vivo (Fig. 4A, B), and expression analysis revealed

584 that $L s B P$ was highly expressed in seeds and showed a temporally overlapping 585 expression pattern with LsAP2 (Fig. 4C; Supplementary Fig. S6).

586 As LsAP2 directly interacted with LsBP at the protein level, and the expression of 587 LsBP gene was also decreased in Lsap2 mutant seeds (Fig. 5A), we further performed 588 dual-luciferase reporter assays to explore the regulatory network between LsAP2 and 589 LsBP. Our results indirectly demonstrated that the protein interaction between LsAP2 590 and LsBP increased the gene expression of LsBP (Fig. 5C). Together, the high 591 biological relevance between $L s A P 2$ and $L s B P$ suggests that LsAP2 may cooperate 592 with LsBP to regulate seed shape in lettuce.

\section{LsAP2 may negatively regulate BR biosynthesis and signaling in seeds}

Previous studies showed that BR plays crucial roles in regulating seed size and seed shape (Jiang et al., 2013; Song, 2017). BR functions through the cell surface receptor BRASSINOSTEROID INSENSITIVE1 (BRI1) and the transcription factor BRASSINAZOLE-RESISTANT1 (BZR1) to control BR-responsive genes (Nolan et al., 2020). In both Arabidopsis and rice, inactivation of BR biosynthesis genes or BR receptors lead to reduced seed size and abnormal seed shape, while elevated BR content or enhanced BR signaling results in increased seed size. In Arabidopsis, the seeds of the BR-deficient mutant de-etiolated2 (det2) and the BR-insensitive mutant bril-5 are smaller and less elongated than those of the WT (Jiang et al., 2013). A rice gain-of-function mutant, slender grain Dominant (slg-D), shows increased BR level and longer grains (Feng et al., 2016), while the loss of function of OsBAK1 (Yuan et $a l .$, 2017), a receptor kinase involved in BR signaling, results in reduced grain length. In this study, we found that the BR content of the Lsap2 mutant seeds was much higher than that of the WT (Fig. 6B), and the transcription level of LsBAKl increased significantly in Lsap2 mutant seeds (Fig. 6C, D). Meanwhile, the seed length and seed length-width ratio of Lsap2 mutant plants were larger than those of the WT (Fig. 2H;

610 Fig. 3E). Our results are consistent with the reports that elevated BR content or 
611 enhanced BR signaling results in increased seed length. Thus, we speculate that

612 LsAP2 may negatively regulate BR biosynthesis and signaling to modulate seed 613 morphology.

614 Taken together, LsAP2 has a significant function during seed development in 615 lettuce, and we proposed a hypothetical working model in which LsAP2 may regulate 616 lettuce seed shape via interacting with LsBP and repressing BR biosynthesis and 617 signaling (Fig. 7). Applying the CRISPR/Cas9 system to generate the knockout plants 618 of the identified interactors or downstream genes of LsAP2 will shed light on the 619 unique regulatory networks during lettuce seed development.

620

621

622

623

624

625

626

627

628

629

630

631

632

633

634

635

636

637

638

639 


\section{Supplementary data}

641 Supplementary data are available at $J X B$ online.

642 Fig. S1. Phylogenetic tree of the euAP2 subfamily.

643 Fig. S2. Expression of $L s A P 2$ in different tissues.

644 Fig. S3. Sanger sequencing analyses of the mutant alleles in T0 generation plants.

645 Fig. S4. Sequencing chromatogram analyses of the edited sites in T0 generation 646 plants.

647 Fig. S5. Scanning electron microscope observation of the seeds from WT and Lsap2 648 mutant plants.

649 Fig. S6. Expression analyses of the interactors of LsAP2.

650 Table S1. Gene information in this study.

651 Table S2. Primers used in this study.

652 Table S3. FPKM values of lettuce AP2 genes at different seed development stages.

653 Table S4. Editing efficiency and mutation types of CRISPR/Cas9-mediated editing of $654 L s A P 2$ in T0 generation plants.

655 Table S5. Summary of the putative off-target sites analyses.

656 Table S6. List of DEGs between WT and Lsap2 mutant plants.

657 Data S1. Coding sequences and amino acid sequences of LsAP2 gene in WT and three 658 mutant lines. 


\section{Acknowledgments}

670 We thank Dr. Shuangxi Fan and Dr. Yingyan Han (Beijing University of Agriculture)

671 for providing the experimental materials. We are grateful to Dr. Xiaolan Zhang (China

672 Agricultural University) for technical assistance. This work was supported by Beijing

673 Leafy Vegetables Innovation Team of Modern Agro-industry Technology Research

674 System (BAIC07-2020) and The Construction of Beijing Science and Technology

675 Innovation and Service Capacity in Top Subjects (CEFF-PXM2019_014207_000032).

\section{Author contributions}

678 QW and CL designed the project. CL, SW, KN, ZC, YW, JY and MQ performed the 679 experiments. CL, SW and KN analyzed the experimental data. QW and CL wrote the 680 manuscript with the help of SW, KN and ZC. All the authors read and approved the 681 final manuscript.

682

683 Data availability statement

684 All data supporting the findings of this study are available within the paper and within 685 its supplementary data published online. 


\section{References}

Alonso-Cantabrana H, Ripoll JJ, Ochando I, Vera A, Ferrándiz C, Martínez-Laborda A. 2007. Common regulatory networks in leaf and fruit patterning revealed by mutations in the Arabidopsis ASYMMETRIC LEAVES1 gene. Development 134, 2663-2671.

Anders S, Huber W. 2010. Differential expression analysis for sequence count data. Genome Biology 11, R106.

Aukerman MJ, Sakai H. 2003. Regulation of flowering time and floral organ identity by a MicroRNA and its APETALA2-like target genes. The Plant Cell 15, 2730-2741.

Bae S, Park J, Kim JS. 2014. Cas-OFFinder: a fast and versatile algorithm that searches for potential off-target sites of Cas9 RNA-guided endonucleases. Bioinformatics 30, 1473-1475.

Botella JR. 2019. Now for the hard ones: is there a limit on CRISPR genome editing in crops? Journal of Experimental Botany 70, 734-737.

Bowman JL, Smyth DR, Meyerowitz EM. 1991. Genetic interactions among floral homeotic genes of Arabidopsis. Development 112, 1-20.

Chen H, Zou Y, Shang Y, Lin H, Wang Y, Cai R, Tang X, Zhou J-M. 2008. Firefly luciferase complementation imaging assay for protein-protein interactions in plants. Plant Physiology 146, 368-376.

Chen K, Wang Y, Zhang R, Zhang H, Gao C. 2019. CRISPR/Cas genome editing and precision plant breeding in agriculture. Annual Review of Plant Biology 70, 667-697.

Chen X. 2004. A microRNA as a translational repressor of APETALA2 in Arabidopsis flower development. Science 303, 2022-2025.

Chen Z, Han Y, Ning K, et al. 2017. Inflorescence development and the role of LsFT in regulating bolting in lettuce (Lactuca sativa L.). Frontiers in Plant Science 8, 2248.

Coen ES, Meyerowitz EM. 1991. The war of the whorls: genetic interactions controlling flower development. Nature 353, 31-37.

Cong L, Ran FA, Cox D, et al. 2013. Multiplex genome engineering using CRISPR/Cas systems. Science 339, 819-823.

Ding L, Yan S, Jiang L, Zhao W, Ning K, Zhao J, Liu X, Zhang J, Wang Q, Zhang X. 2015. HANABA TARANU (HAN) bridges meristem and organ primordia boundaries through PINHEAD, JAGGED, BLADE-ON-PETIOLE2 and CYTOKININ OXIDASE 3 during flower development in Arabidopsis. PLoS Genetics 11, e1005479.

Drews GN, Bowman JL, Meyerowitz EM. 1991. Negative regulation of the Arabidopsis homeotic gene AGAMOUS by the APETALA2 product. Cell 65, 991-1002.

Feng Z, Wu C, Wang C, et al. 2016. SLG controls grain size and leaf angle by modulating brassinosteroid homeostasis in rice. Journal of Experimental Botany 67, 4241-4253.

Figueiredo DD, Köhler C. 2018. Auxin: a molecular trigger of seed development. Genes \& Development 32, 479-490. Han Y, Chen Z, Lv S, Ning K, Ji X, Liu X, Wang Q, Liu R, Fan S, Zhang X. 2016. MADS-box genes and gibberellins regulate bolting in lettuce (Lactuca sativa L.). Frontiers in Plant Science 7, 1889.

Hellens RP, Allan AC, Friel EN, Bolitho K, Grafton K, Templeton MD, Karunairetnam S, Gleave AP, Laing WA. 2005. Transient expression vectors for functional genomics, quantification of promoter activity and RNA silencing in plants. Plant Methods 1, 13.

Iwakawa H, Ueno Y, Semiarti E, et al. 2002. The ASYMMETRIC LEAVES2 gene of Arabidopsis thaliana, required for formation of a symmetric flat leaf lamina, encodes a member of a novel family of proteins characterized by cysteine repeats and a leucine zipper. Plant \& Cell Physiology 43, 467-478.

Jiang H, Köhler C. 2012. Evolution, function, and regulation of genomic imprinting in plant seed development. Journal of Experimental Botany 63, 4713-4722.

Jiang L, Ma X, Zhao S, et al. 2019. The APETALA2-like transcription factor SUPERNUMERARY BRACT controls rice seed shattering and seed size. The Plant Cell 31, 17-36.

Jiang WB, Huang HY, Hu YW, Zhu SW, Wang ZY, Lin WH. 2013. Brassinosteroid regulates seed size and shape in Arabidopsis. Plant Physiology 162, 1965-1977.

Jofuku KD, den Boer BG, Van Montagu M, Okamuro JK. 1994. Control of Arabidopsis flower and seed development by the homeotic gene APETALA2. The Plant Cell 6, 1211-1225.

Jofuku KD, Omidyar PK, Gee Z, Okamuro JK. 2005. Control of seed mass and seed yield by the floral homeotic gene APETALA2. Proceedings of the National Academy of Sciences, USA 102, 3117-3122. 
Kim D, Langmead B, Salzberg SL. 2015. HISAT: a fast spliced aligner with low memory requirements. Nature Methods 12, 357-360.

Kim S, Soltis PS, Wall K, Soltis DE. 2006. Phylogeny and domain evolution in the APETALA2-like gene family. Molecular Biology and Evolution 23, 107-120.

Kumar V, Jain M. 2015. The CRISPR-Cas system for plant genome editing: advances and opportunities. Journal of Experimental Botany 66, 47-57.

Kunst L, Klenz JE, Martinez-Zapater J, Haughn GW. 1989. AP2 gene determines the identity of perianth organs in flowers of Arabidopsis thaliana. The Plant Cell 1, 1195-1208.

Larkin MA, Blackshields G, Brown NP, et al. 2007. Clustal W and Clustal X version 2.0. Bioinformatics 23, 2947-2948.

Lee DY, Lee J, Moon S, Park SY, An G. 2007. The rice heterochronic gene SUPERNUMERARY BRACT regulates the transition from spikelet meristem to floral meristem. The Plant Journal 49, 64-78.

Leon-Kloosterziel KM, Keijzer CJ, Koornneef M. 1994. A seed shape mutant of Arabidopsis that is affected in integument development. The Plant Cell 6, 385-392.

Li J, Wen J, Lease KA, Doke JT, Tax FE, Walker JC. 2002. BAK1, an Arabidopsis LRR receptor-like protein kinase, interacts with BRI1 and modulates brassinosteroid signaling. Cell 110, 213-222.

Li N, Li Y. 2015. Maternal control of seed size in plants. Journal of Experimental Botany 66, 1087-1097.

Li N, Xu R, Li Y. 2019. Molecular networks of seed size control in plants. Annual Review of Plant Biology 70, 435-463. Liu H, Ding Y, Zhou Y, Jin W, Xie K, Chen LL. 2017. CRISPR-P 2.0: an improved CRISPR-Cas9 tool for genome editing in plants. Molecular Plant 10, 530-532.

Livak KJ, Schmittgen TD. 2001. Analysis of relative gene expression data using real-time quantitative PCR and the 2(-Delta Delta C(T)) Method. Methods 25, 402-408.

Ma X, Feng F, Zhang Y, et al. 2019. A novel rice grain size gene OsSNB was identified by genome-wide association study in natural population. PLoS Genetics 15, e1008191.

Maes T, Van de Steene N, Zethof J, Karimi M, D'Hauw M, Mares G, Van Montagu M, Gerats T. 2001. Petunia Ap2-like genes and their role in flower and seed development. The Plant Cell 13, 229-244.

Mao X, Cai T, Olyarchuk JG, Wei L. 2005. Automated genome annotation and pathway identification using the KEGG Orthology (KO) as a controlled vocabulary. Bioinformatics 21, 3787-3793.

McAbee JM, Hill TA, Skinner DJ, Izhaki A, Hauser BA, Meister RJ, Venugopala Reddy G, Meyerowitz EM, Bowman JL, Gasser CS. 2006. ABERRANT TESTA SHAPE encodes a KANADI family member, linking polarity determination to separation and growth of Arabidopsis ovule integuments. The Plant Journal 46, 522-531.

Moles AT, Ackerly DD, Webb CO, Tweddle JC, Dickie JB, Westoby M. 2005. A brief history of seed size. Science 307, 576-580.

Morel P, Heijmans K, Rozier F, Zethof J, Chamot S, Bento SR, Vialette-Guiraud A, Chambrier P, Trehin C, Vandenbussche M. 2017. Divergence of the floral A-Function between an asterid and a rosid species. The Plant Cell 29, 1605-1621.

Ning K, Han Y, Chen Z, Luo C, Wang S, Zhang W, Li L, Zhang X, Fan S, Wang Q. 2019. Genome-wide analysis of MADS-box family genes during flower development in lettuce. Plant, Cell \& Environment 42, 1868-1881.

Nolan TM, Vukasinovic N, Liu D, Russinova E, Yin Y. 2020. Brassinosteroids: multidimensional regulators of plant growth, development, and stress responses. The Plant Cell 32, 295-318.

Ohto MA, Fischer RL, Goldberg RB, Nakamura K, Harada JJ. 2005. Control of seed mass by APETALA2. Proceedings of the National Academy of Sciences, USA 102, 3123-3128.

Ohto MA, Floyd SK, Fischer RL, Goldberg RB, Harada JJ. 2009. Effects of APETALA2 on embryo, endosperm, and seed coat development determine seed size in Arabidopsis. Sexual Plant Reproduction 22, 277-289.

Park J, Bae S, Kim JS. 2015. Cas-Designer: a web-based tool for choice of CRISPR-Cas 9 target sites. Bioinformatics 31, 4014-4016.

Reyes-Chin-Wo S, Wang Z, Yang X, et al. 2017. Genome assembly with in vitro proximity ligation data and whole-genome triplication in lettuce. Nature Communications 8, 14953.

Ripoll JJ, Roeder AH, Ditta GS, Yanofsky MF. 2011. A novel role for the floral homeotic gene APETALA2 during Arabidopsis fruit development. Development 138, 5167-5176.

Sgamma T, Pape J, Massiah A, Jackson S. 2016. Selection of reference genes for diurnal and developmental time-course real-time PCR expression analyses in lettuce. Plant Methods 12, 21.

Shan QW, Wang YP, Li J, et al. 2013. Targeted genome modification of crop plants using a CRISPR-Cas system. 
Nature Biotechnology 31, 686-688.

Song XJ. 2017. Crop Seed Size: BR Matters. Molecular Plant 10, 668-669.

Sreenivasulu N, Wobus U. 2013. Seed-development programs: a systems biology-based comparison between dicots and monocots. Annual Review of Plant Biology 64, 189-217.

Steinbrecher T, Leubner-Metzger G. 2017. The biomechanics of seed germination. Journal of Experimental Botany 68, 765-783.

Tamura K, Peterson D, Peterson N, Stecher G, Nei M, Kumar S. 2011. MEGA5: molecular evolutionary genetics analysis using maximum likelihood, evolutionary distance, and maximum parsimony methods. Molecular Biology and Evolution 28, 2731-2739.

Venglat SP, Dumonceaux T, Rozwadowski K, Parnell L, Babic V, Keller W, Martienssen R, Selvaraj G, Datla R. 2002. The homeobox gene BREVIPEDICELLUS is a key regulator of inflorescence architecture in Arabidopsis. Proceedings of the National Academy of Sciences, USA 99, 4730-4735.

Wang Y, Li B, Du M, Eneji AE, Wang B, Duan L, Li Z, Tian X. 2012. Mechanism of phytohormone involvement in feedback regulation of cotton leaf senescence induced by potassium deficiency. Journal of Experimental Botany $\mathbf{6 3}$, 5887-5901.

Xing HL, Dong L, Wang ZP, Zhang HY, Han CY, Liu B, Wang XC, Chen QJ. 2014. A CRISPR/Cas9 toolkit for multiplex genome editing in plants. BMC Plant Biology 14, 327.

Young MD, Wakefield MJ, Smyth GK, Oshlack A. 2010. Gene ontology analysis for RNA-seq: accounting for selection bias. Genome Biology 11, R14.

Yuan H, Fan S, Huang J, et al. 2017. 08SG2/OsBAK1 regulates grain size and number, and functions differently in Indica and Japonica backgrounds in rice. Rice 10, 25.

Zhang L, Su W, Tao R, et al. 2017. RNA sequencing provides insights into the evolution of lettuce and the regulation of flavonoid biosynthesis. Nature Communications 8, 2264.

Zhao J, Li G, Yi G-X, Wang B-M, Deng A-X, Nan T-G, Li Z-H, Li QX. 2006. Comparison between conventional indirect competitive enzyme-linked immunosorbent assay (icELISA) and simplified icELISA for small molecules. Analytica Chimica Acta 571, 79-85.

Zumajo-Cardona C, Pabon-Mora N. 2016. Evolution of the APETALA2 gene lineage in seed plants. Molecular Biology and Evolution 33, 1818-1832. 


\section{Figure legends}

Fig. 1. Expression pattern of $L \boldsymbol{S A P 2}$. (A) Different seed development stages. S0, the day of pollination; S1-S9, 1-9 days after pollination (DAP), respectively. (B) Expression of $L S A P 2$ in floral organs. br, bract; pe, petal; st, stamen; pi, pistil; se, seed. Values are means $\pm \mathrm{SD}(n=3)$. (C) Expression of $L s A P 2$ at different seed development stages. Values are means $\pm \mathrm{SD}(n=3)$. The expression data were normalized using $L s P P 2 A-1$ and LsTIP41 as reference genes. (D) The capitulum of WT at S0 stage. (E) A floret in D. pt, petal tube. (F) The capitulum of $p L s A P 2: G U S$ plant at S0 stage. (G) A floret in F. (H) The seed head of pLsAP2:GUS plant at S3 stage. pa, pappus; be, beak. (I) The seed of WT at S0 stage. (J-M) The seeds of pLsAP2:GUS plants at S0, S1, S2, and S3 stages, respectively. (N) Longitudinal section of the seed from WT. sc, seed coat (pericarp and integuments). (O) Longitudinal section of the seed from $p L s A P 2: G U S$ plant. (P) Magnified image of the longitudinal section in O. (Q) Transverse section of the seed from pLsAP2:GUS plant. Scale bars: $1 \mathrm{~mm}$.

Fig. 2. CRISPR/Cas9-mediated editing of LsAP2 alters seed shape. (A) Gene structure of $L s A P 2$. The two target sites are indicated by arrows. Rectangles and lines represent exons and introns, respectively. Red regions indicate the two AP2 domains. Green region indicates the miR172 binding site. Blue region indicates the untranslated region. (B) Sanger sequencing analyses of the mutant alleles in T1 homozygous plants. The target sequences are indicated under the black line. The PAM sequences are depicted in red. The insertion is depicted in green. Numbers on the right side indicate the deletion (-) or insertion (+) compared with WT. (C) Sequencing chromatogram examples of the edited site in the mutant allele compared with WT. Arrow indicates the 1-bp insertion. (D-F) Seed phenotype of WT and three Lsap2 mutant lines. Extended tips are marked with dotted boxes in D. (G) Seed germination tests of WT and Lsap2 mutant plants. (H) Seed length of WT and Lsap2 mutant plants. Values are means \pm SD $(n=10)$. (I) Seed width of WT and Lsap2 mutant plants. Values are 
means $\pm \mathrm{SD}(n=10)$. (J) The 1,000-grain weight of WT and Lsap2 mutant plants. Values are means $\pm \mathrm{SD}(n=5)$. (K) Seed germination rate of WT and Lsap2 mutant plants. Values are means $\pm \operatorname{SD}(n=3)$. Significant difference analysis was conducted with two-tailed Student's $t$ tests (ns, not significant; **, $P<0.01$ ). Scale bars: $2 \mathrm{~mm}$.

Fig. 3. Important roles of $L s A P 2$ in regulating seed morphology. (A-D) Seed phenotype of WT and three Lsap2 mutant lines at different seed development stages. Upper and lower brackets indicate beaks and seeds, respectively. Asterisks indicate the junctions between beaks and seeds. (A) The seeds of WT and Lsap2 mutant plants, 0 DAP. (B) The seeds of WT and Lsap2 mutant plants, 1 DAP. (C) The seeds of WT and Lsap2 mutant plants, 2 DAP. (D) The seeds of WT and Lsap2 mutant plants, 20 DAP. (E) Seed length-width ratio of WT and Lsap2 mutant plants. Values are means \pm SD $(n=10)$. (F) Seed beak diameter of WT and Lsap2 mutant plants. Values are means \pm SD $(n=10)$. (G) The BTS between beak and seed of WT and Lsap2 mutant plants. Values are means \pm SD $(n=10)$. Significant difference analysis was conducted with two-tailed Student's $t$ tests $(* *, P<0.01)$. Scale bars: $1 \mathrm{~mm}$.

\section{Fig. 4. LsAP2-related protein interaction networks during lettuce seed} development. (A) LsAP2 interacts with LsAS2, LsBP, LsKAN1, and LsKAN2 in yeast two-hybrid assays. AD, activation domain; BD, binding domain. (B) LCI assays show that LsAP2 interacts with LsAS2, LsBP, and LsKAN1 in planta. (C) Expression of $L s B P$ and $L s K A N 1$ at different seed development stages. Values are means \pm SD ( $n$ =3). The expression data were normalized using $L s P P 2 A-1$ and $L s T I P 41$ as reference genes.

Fig. 5. LsAP2 affects the expression of fruit development related genes. (A) Expression of $L s B P$ and $L s R P L$ between WT and Lsap2 mutant plants. Values are means $\pm \mathrm{SD}(n=3)$. The expression data were normalized using LsPP2A-1 and LSTIP41 as reference genes. (B) Schematic diagram of the reporter and effector constructs. (C) Dual-luciferase reporter assays show the interactions of LsAP2 and 
LsBP with the $L s B P$ promoter. Values are means \pm SD $(n=6)$. Significant difference analysis was conducted with two-tailed Student's $t$ tests (ns, not significant; ${ }^{*}, P<$ $0.05 ; * *, P<0.01)$.

Fig. 6. Identification of differentially expressed genes between WT and Lsap2 mutant plants. (A) GO enrichment analysis of the DEGs. (B) The IAA and BR levels in the developing seeds of WT and Lsap2 mutant plants. Values are means $\pm \operatorname{SD}(n=$ 3). (C) Heat map for the expression levels of BR-related genes between WT and Lsap2 mutant plants. Red boxes indicate up-regulation, and blue boxes indicate down-regulation. (D) Expression analyses of BR-related genes between WT and Lsap2 mutant plants. Values are means $\pm \mathrm{SD}(n=3)$. The expression data were normalized using $L s P P 2 A-1$ and LsTIP4I as reference genes. Significant difference analysis was conducted with two-tailed Student's $t$ tests (ns, not significant; *, $P<$ $0.05 ; * *, P<0.01)$.

Fig. 7. A hypothetical working model of LsAP2 in regulating seed shape in lettuce. LsAP2 may regulate seed shape by directly interacting with LsBP and promoting the expression of $L s B P$. Meanwhile, LsAP2 may negatively regulate BR biosynthesis and signaling in lettuce seeds. The biological function of $L s B P$ needs to be further confirmed in lettuce. Arrow, positive regulation; bar, negative regulation. 


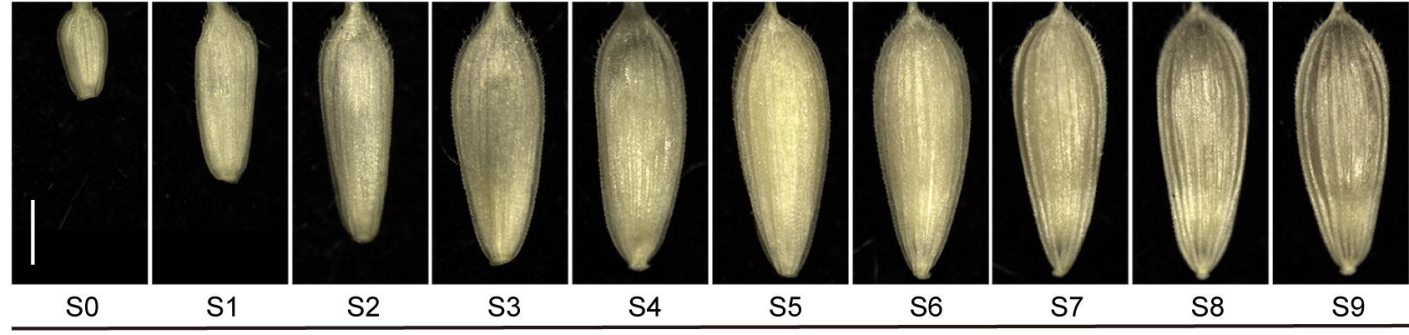

Different seed development stages

B

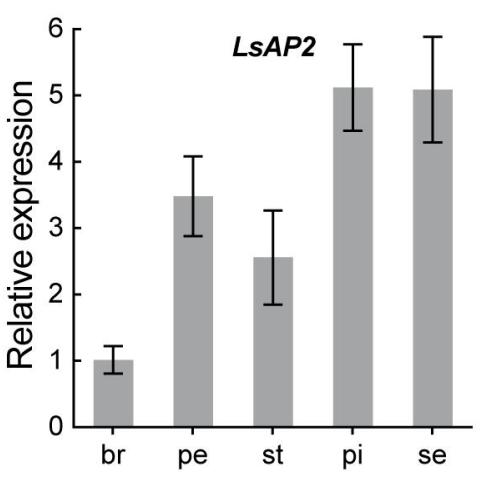

D
E $\quad$ pe $F$

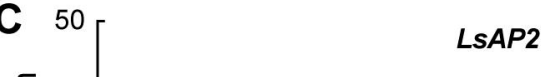

F

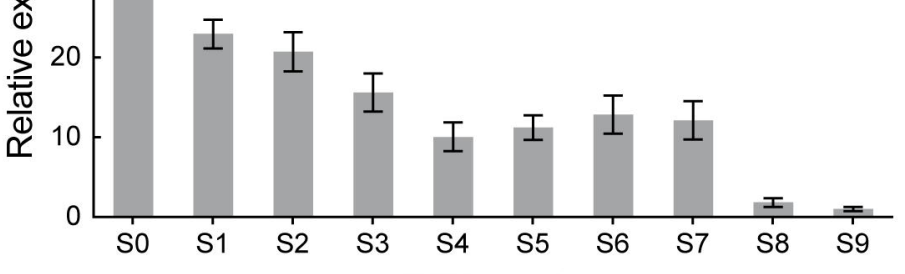




\section{Target 1 Target 2}
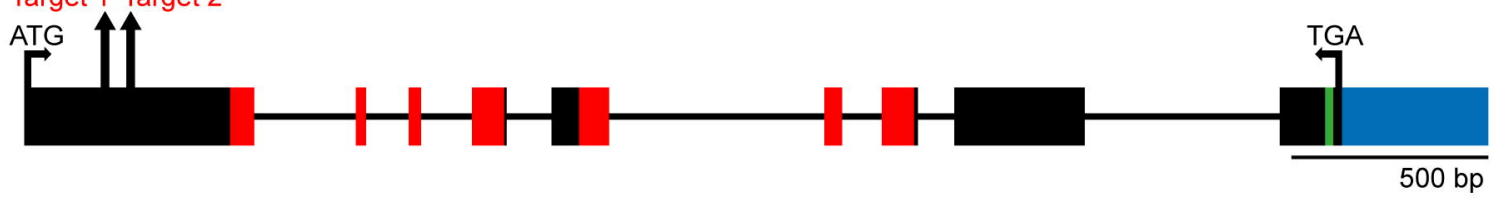

Target 1

PAM

PAM

Target 2

$\overline{\text { AAGCACTACAGGTGATGGGAGGTTGTTTGGTTTTTCTATGACGGAAAACTCCTCTTGGGACAGCGATCCTCC }}$ WT (0) AAGCACTACAGGTGAT $\ldots \ldots \ldots$ AAGCACTACAGGTGATTGGGAGGTTGTTTGGTTTTTCTATGACGGAAAACTCCTCTTGGGACAGCGATCCTCC \#49 $(+1,0)$ AAGCACTACA-- - - TGGGAGGTTGTTTGGTTTTTCTATGACGGAAAACTCCTCTTGGGACAGCGATCCTCC \#100 $(-5,0)$

C

Target 1

PAM

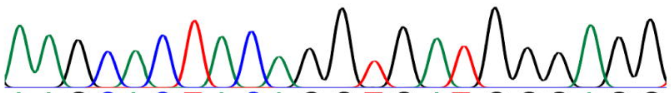
WT A A G C C T A CA G G T A T G GAGG
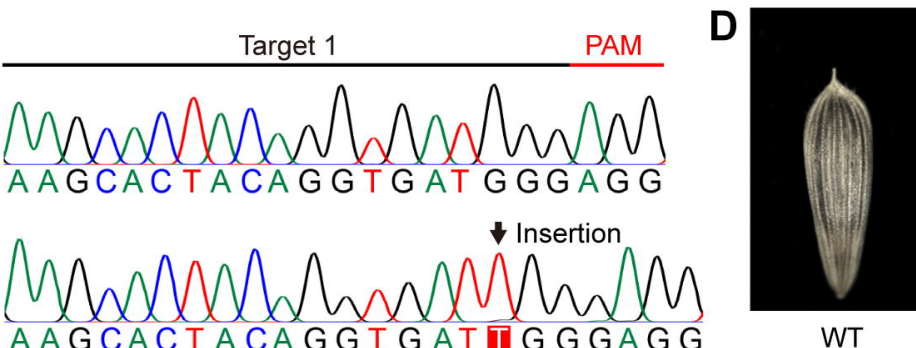

WT
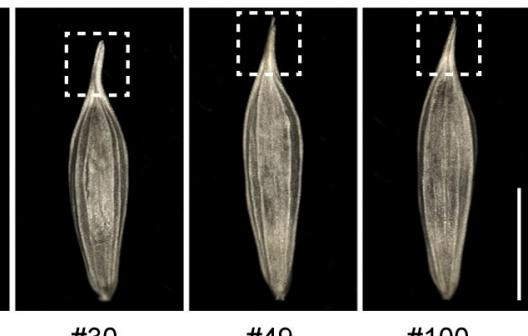
\#49 A A G C A C T A CA G G T G AT T G G G A G

E
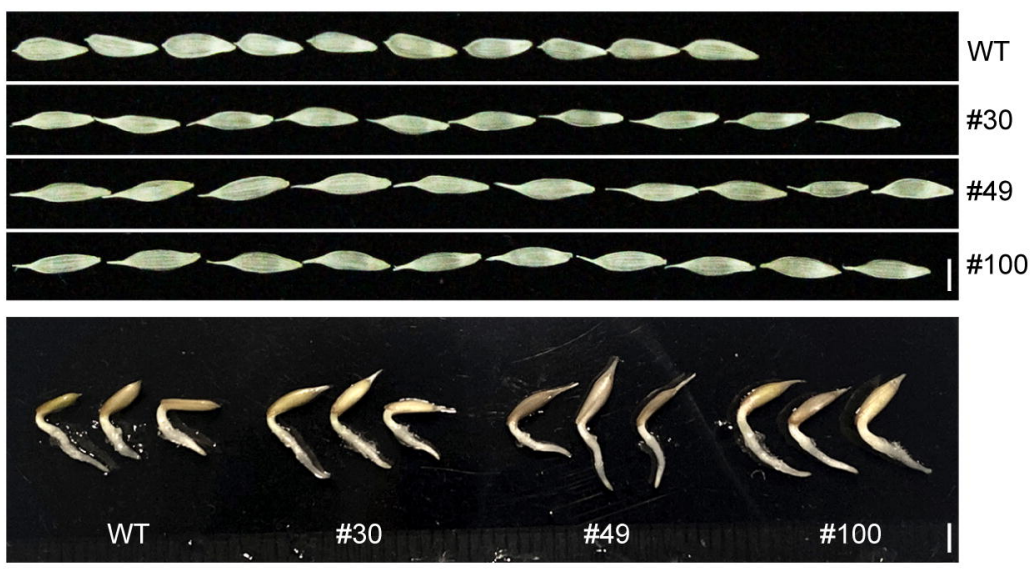

\#30

\#49

\#100

LsAP2-CRISPR/Cas9

$\mathbf{F}$
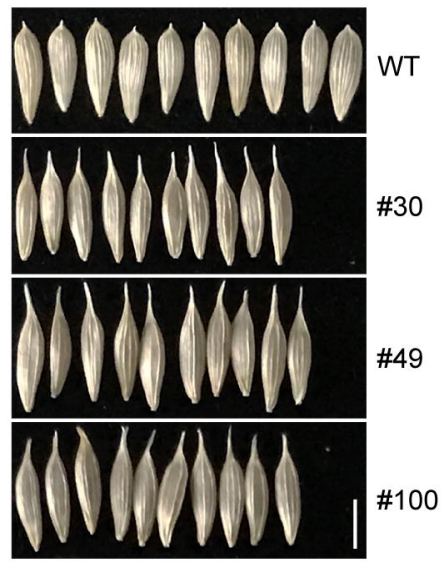

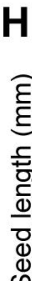
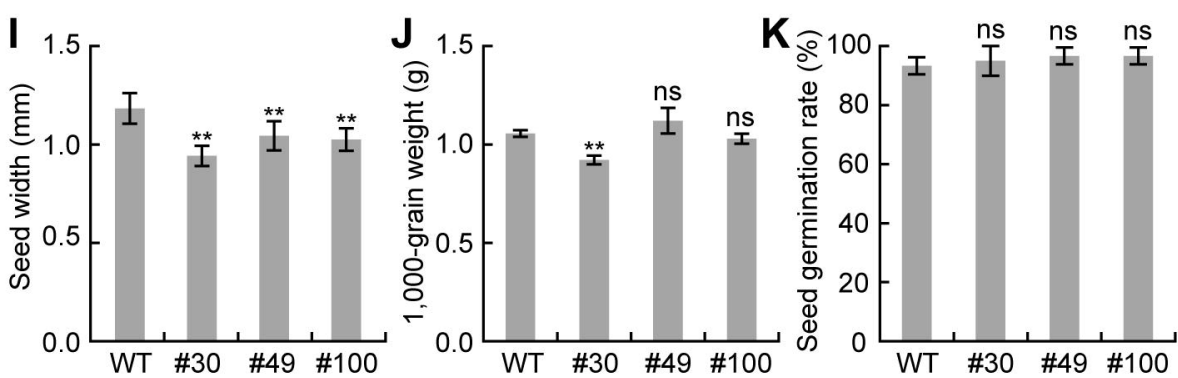
AD

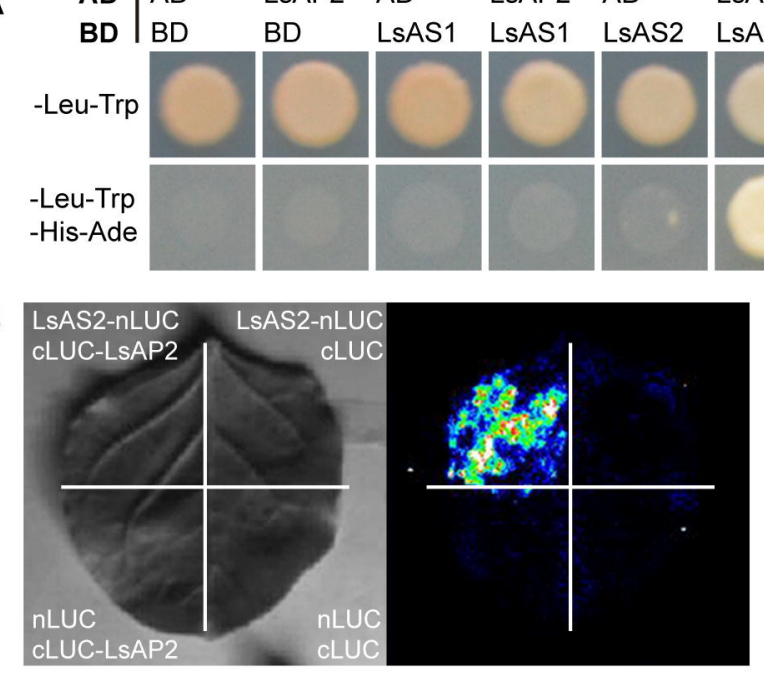

LSKAN1-nLUC LsKAN1-nLUC
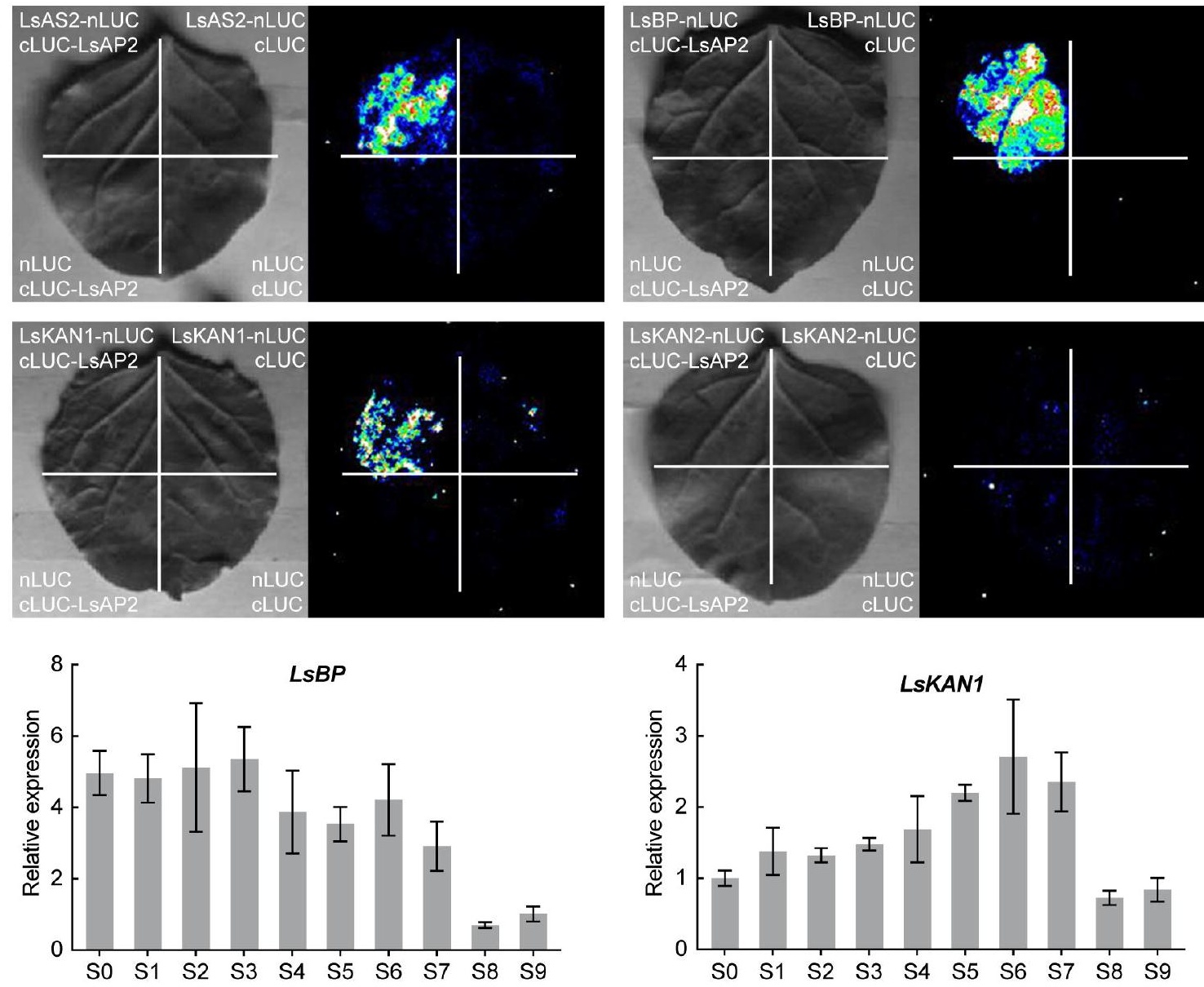


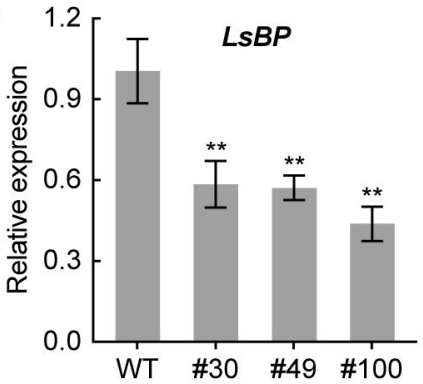

Reporter:

-CaMV 35S - REN - $-p L s B P$ - LUC CaMV tem

CaMV tem

Effector:

-CaMV 35S - CaMV tem

-CaMV 35S - LsAP2 - CaMV tem

-CaMV 35S - LsBP - CaMV tem

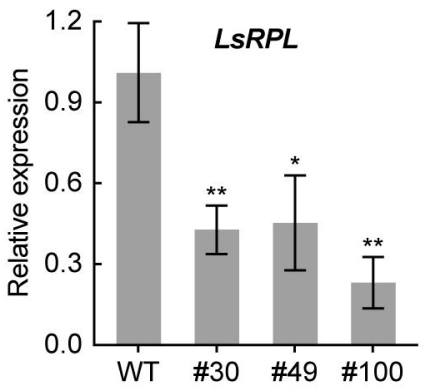

C

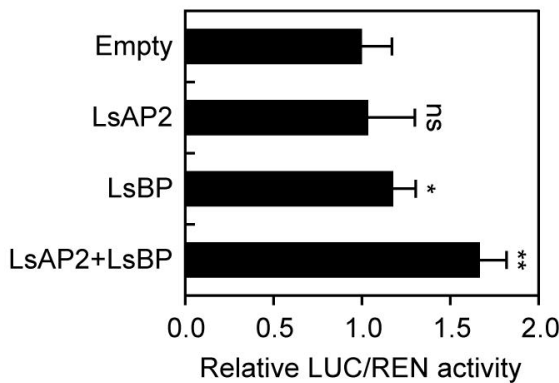




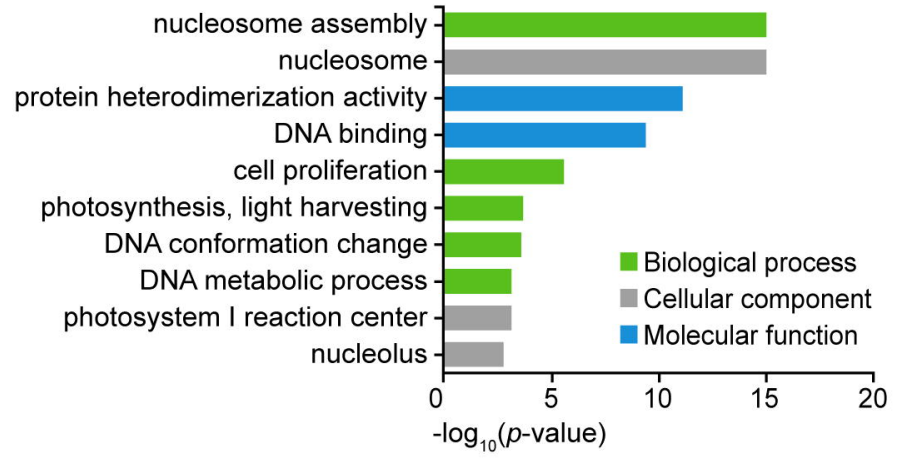

B

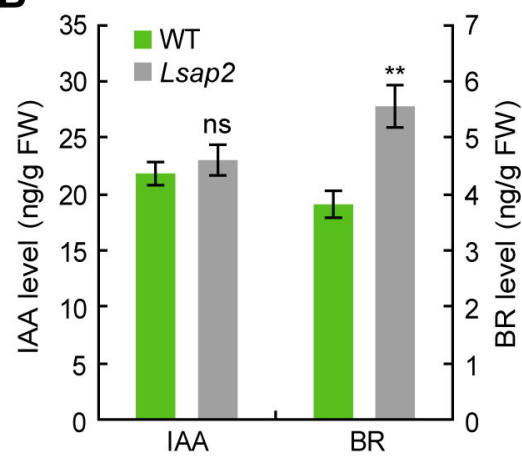

D
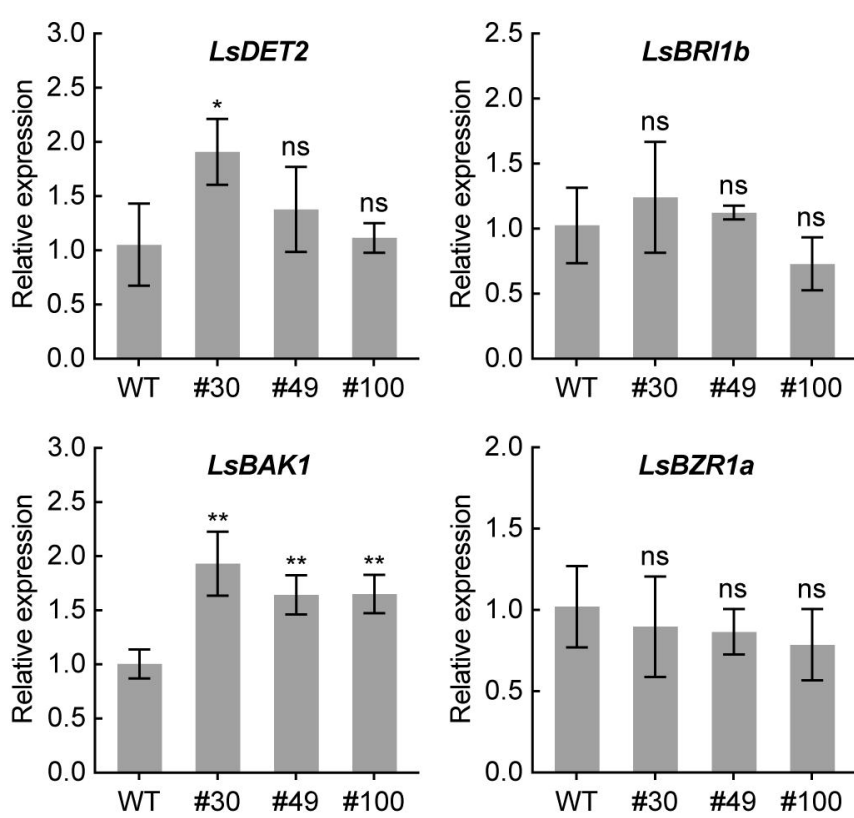

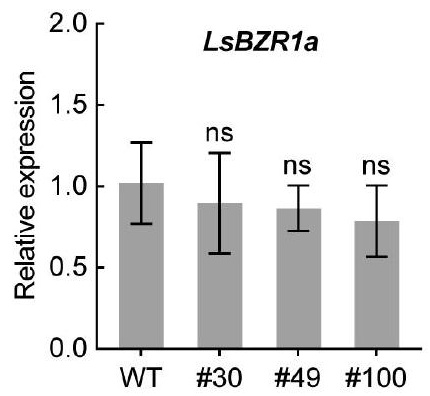




\section{LsBP}

\section{LsAP2}

$\perp$

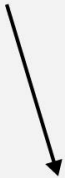

BR biosynthesis and signaling

\section{seed shape}

